\title{
HOMOLOGY IN VARIETIES OF GROUPS. II
}

\author{
BY \\ C. R. LEEDHAM-GREEN
}

\begin{abstract}
The study of (co-) homology groups $\mathfrak{B}_{n}(\Pi, A), \mathfrak{B}^{n}(\Pi, A), \mathfrak{B}$ a variety, $\Pi$ a group in $\mathfrak{B}$, and $A$ a suitable $\Pi$-module, is pursued. They are compared with a certain Tor and Ext. The definition of the homology of an epimorphism due to Rinehart is shown to agree with that due to Barr and Beck (whenever both are defined). The edge effects of a spectral sequence are calculated.
\end{abstract}

Introduction. In a previous paper [21], henceforth referred to as [HI], we discussed a homology and cohomology theory for a group $\Pi$ with respect to a variety $\mathfrak{B}$ containing $\Pi$, and showed that the theory does not always agree with the appropriate Tor and Ext. We pursue the matter with special attention to modules on which the group acts trivially. In dimension 1 the problem is related to the dimension problem (see the beginning of \$2). Two results of more general homological interest are produced: in $\S 1$ we prove that Rinehart's definition of the homology of an epimorphism agrees with that of Barr and Beck (whenever both are defined), and in $\$ 3$ we calculate the edge effects of a well-known universal coefficient spectral sequence.

Preliminaries. The principal conventions and definitions of $[\mathrm{HI}]$ will be used. In particular, $\mathfrak{B}$ denotes a variety containing the group $\Pi ; \mathfrak{B} \Pi$ is a quotient ring of the integral group ring $Z \Pi$ obtained by dividing out by the images of the Fox derivatives of the words defining $\mathfrak{B}$, as in [HI, §2]; if $A$ is a $\mathfrak{B} \Pi$-module, then $\mathfrak{B}_{n}(\Pi, A)$ and $\mathfrak{B}^{n}(\Pi, A)$ are the triple (co-) homology groups defined in [HI, §3]; if $\Gamma \rightarrow \Pi \in|(\mathfrak{B}, \Pi)|, D \Gamma$ is a right $\mathfrak{B} \Pi$-module which represents $\operatorname{Der}(\Gamma,-)$ as a functor from right $\mathfrak{B} \Pi$-modules to $\boldsymbol{A b}$. In addition to the conventions listed in the preliminaries to $[\mathrm{HI}], \mathfrak{R}_{c}$ will denote the variety of nilpotent groups of class at most $c$, and $\mathbb{E}$ the variety of trivial groups. Thus $[\mathfrak{E}, \mathfrak{B}]$ is the variety "centre by $\mathfrak{B}$ ". If $\mathfrak{u}$ is a variety, $\mathfrak{u}\langle\Pi\rangle$ denotes the verbal subgroup of $\Pi$ defined by $\mathfrak{u}$.

1. Exact sequences. If $\alpha: \Gamma_{0} \rightarrow \Gamma_{1}$ is a surjection in $(\mathfrak{B}, \Pi)$ and $A$ is a $\mathfrak{B} \Pi$ module, then there is an exact sequence

$$
\begin{aligned}
\cdots \rightarrow \mathfrak{B}_{n}\left(\Gamma_{0}, A\right) \rightarrow \mathfrak{B}_{n}\left(\Gamma_{1}, A\right) & \rightarrow M_{n-1}(\alpha, A) \rightarrow \\
\cdots & \rightarrow M_{0}(\alpha, A) \rightarrow \mathfrak{B}_{0}\left(\Gamma_{0}, A\right) \rightarrow \mathfrak{B}_{0}\left(\Gamma_{1}, A\right) \rightarrow 0
\end{aligned}
$$

Received by the editors September 10, 1970.

AMS 1970 subject classifications. Primary 18H40, 20E10; Secondary 18C15, 18G10, 18H40, 18H10, $20 \mathrm{J05}$.

Key words and phrases. Homology in varieties of groups, universal coefficients, spectral sequence, Rinehart's exact sequence.

Copyright (C) 1972, American Mathematical Society 
due to Rinehart which appears in [HI] as $(\hat{2} .1)$. The map of $\mathfrak{B}_{n}\left(\Gamma_{0}, A\right)$ into $\mathfrak{B}_{n}\left(\Gamma_{1}, A\right)$ is $\mathfrak{B}_{n}(\alpha, A)$. We produce a slightly more concrete construction of the functors $M_{n}(\alpha, A)$ than Rinehart's, linking them with the triple theory. The reader who is not familiar with Rinehart's paper [13] can use Proposition 1.1 below as a definition of $M_{n}(\alpha, A)$; the present paper and [HI] then become independent of Rinehart's treatment except for the identification of $M_{0}(\alpha, A)$ [HI, Theorem 2.1], which can presumably be deduced directly from Proposition 1.1. Consequently in the proof of the proposition we use the concepts and notations of [13] without explanation. Recall that $\mathfrak{B}_{n}(\Gamma, A)$ may be calculated from the Barr-Beck resolution as the homology of $\cdots \rightarrow \operatorname{Diff}\left(\Gamma B_{n}, A\right) \rightarrow \operatorname{Diff}\left(\Gamma B_{n-1}, A\right) \rightarrow \cdots$ (cf. [HI, §2]).

Proposition 1.1. Let $\alpha: \Gamma_{0} \rightarrow \Gamma_{1}$ be a surjection in $(\mathfrak{B}, \Pi)$ and $A$ be a left $\mathfrak{B} \Pi$ module. Put $L_{n}(\alpha, A)=\operatorname{ker} \operatorname{Diff}\left(\alpha B_{n}, A\right)$ so that $0 \rightarrow \boldsymbol{L}(\alpha, A) \rightarrow \operatorname{Diff}\left(\Gamma_{0} B, A\right)$ $\rightarrow \operatorname{Diff}\left(\Gamma_{1} B, A\right) \rightarrow 0$ is a short exact sequence of complexes. Then $M(\alpha, A)$ is the homology of $\boldsymbol{L}(\alpha, A)$, and the long exact sequence in homology obtained from the above short exact sequence is the sequence (1.1).

Proof. Define $\Lambda_{i}:(\mathfrak{B}, \Pi)^{\prime} \rightarrow(\mathfrak{B}, \Pi)$ by $\left(\Gamma_{0} \rightarrow \Gamma_{1}\right) \Lambda_{i}=\Gamma_{i}, \quad i=0,1$, with the obvious behaviour on morphisms. Then $0 \rightarrow \boldsymbol{L}(-, A) \rightarrow \operatorname{Diff}\left(-\Lambda_{0} \boldsymbol{B}, A\right)$ $\rightarrow \operatorname{Diff}\left(-\Lambda_{1} \boldsymbol{B}, \boldsymbol{A}\right) \rightarrow 0$ is an exact sequence of complexes from $(\mathfrak{B}, \Pi)^{\prime}$ to $\boldsymbol{A b}$. It is enough to prove that the functors appearing in these complexes are flask, and that the complexes are acyclic on projectives. Let $(\mathscr{S}, \Pi)$ be the category of sets over the underlying set of $\Pi$. Regard $(\mathscr{S}, \Pi)$ as a base, with projective class the surjections. In $(\mathscr{S}, \Pi)$ every surjection splits, so every functor from $(\mathscr{S}, \Pi)$ to $\boldsymbol{A b}$ is flask [13, Lemma 2.3]. But the forgetful functor from $(\mathfrak{B}, \Pi)$ to $(\mathscr{S}, \Pi)$ is a morphism of bases and $\operatorname{Diff}\left(-B_{n}, A\right)$ factors through this functor $(n \geqq 0)$, so $\operatorname{Diff}\left(-B_{n}, A\right):(\mathfrak{B}, \Pi) \rightarrow A b$ is a flask functor [13, Remark 2.14]. Also, $\Lambda_{0}$ and $\Lambda_{1}$ are morphisms of bases, so $\operatorname{Diff}\left(-\Lambda_{i} B_{n}, A\right):(\mathfrak{B}, \Pi)^{\prime} \rightarrow A b$ is flask. Hence $L_{n}(-, A)$ is flask as well. Finally, if $\alpha: \Gamma_{0} \rightarrow \Gamma_{1}$ is projective then $\Gamma_{0}$ and $\Gamma_{1}$ are $\mathfrak{B}$-splitting groups and the complexes Diff $\left(\alpha \Lambda_{i} \boldsymbol{B}, A\right)$ are acyclic. Hence $L_{n}(\alpha, A)$ is also acyclic.

REMARK 1.2. If $\alpha$ is a split surjection the sequence (1.1) clearly divides into split short exact sequences $0 \rightarrow M_{n}(\alpha, A) \rightarrow \mathfrak{B}_{n}\left(\Gamma_{0}, A\right) \rightarrow \mathfrak{B}_{n}\left(\Gamma_{1}, A\right) \rightarrow 0$.

REMARK 1.3. The construction of Barr and Beck in [4, §2] gives, for any morphism $\alpha: \Gamma_{0} \rightarrow \Gamma_{1}$ in $(\mathfrak{B}, \Pi)$, homology groups $H_{n} \alpha$ and a long exact sequence

$$
\begin{aligned}
\cdots \rightarrow \mathfrak{B}_{n}\left(\Gamma_{0}, A\right) \rightarrow \mathfrak{B}_{n}\left(\Gamma_{1}, A\right) & \rightarrow H_{n} \alpha \rightarrow \\
\cdots & \rightarrow \mathfrak{B}_{0}\left(\Gamma_{0}, A\right) \rightarrow \mathfrak{B}_{0}\left(\Gamma_{1}, A\right) \rightarrow H_{0} \alpha \rightarrow 0 .
\end{aligned}
$$

This is done by using the mapping cone of the chain transformation $\operatorname{Diff}(\alpha \boldsymbol{B}, A)$. Thus, writing $X_{n}, Y_{n}$ for $\operatorname{Diff}\left(\Gamma_{0} B_{n}, A\right)$, Diff $\left(\Gamma_{1} B_{n}, A\right)$ respectively, $H_{*} \alpha$ is the homology of a complex which is given in dimension $n$ by $Y_{n} \oplus X_{n-1}\left(X_{-1}=0\right)$, and (1.2) comes from a short exact sequence of complexes which in dimension $n$ is $0 \rightarrow Y_{n} \rightarrow Y_{n} \oplus X_{n-1} \rightarrow X_{n-1} \rightarrow 0$, the homomorphisms being natural. Now suppose that $\alpha$ is a surjection, and put $L_{n}=L_{n}(\alpha, A)$ as in the proposition. Then 
there is a commutative diagram of complexes whose "section" in dimension $n$ is

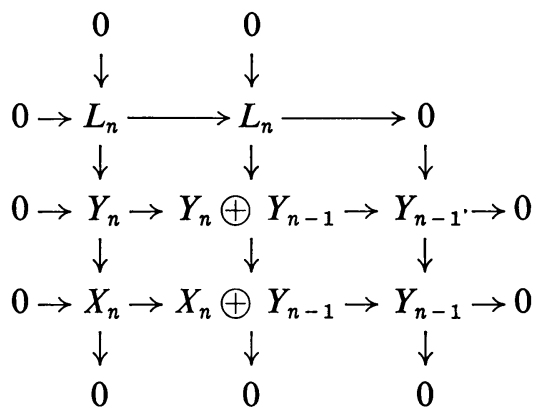

Here the middle complex is the mapping cone of $1_{Y}$, and the homomorphisms are induced in the obvious way by $\alpha$ and various identity maps. The middle row shows that the mapping cone of $1_{Y}$ is acyclic, and the middle column then equates $H_{n} \alpha$ with $H_{n-1} L_{*}=M_{n-1}(\alpha, A)$. Thus if $\alpha$ is a surjection, Rinehart's long exact sequence agrees with that of Barr and Beck. The argument of course carries through in greater generality, in fact whenever the Barr-Beck sequence is defined and $\alpha$ is in the projective class defined by the triple (cf. [13, Remark 5.6]). I am grateful to D. E. Cohen for showing me the above argument.

We now construct a sequence in some way analogous to (1.1) for Tor, and compare the two sequences. If $\Gamma \rightarrow \Pi \in|(\mathfrak{B}, \Pi)|$, recall from [HI] that $D \Gamma$ is defined as I $\otimes_{\mathrm{z} \Gamma} \mathfrak{B} \Pi$. If $P_{*} \rightarrow D \Gamma \rightarrow 0$ is a projective resolution of $D \Gamma$ then since $D\left(\Gamma B_{*}\right) \rightarrow D \Gamma \rightarrow 0$ is also a complex of projective $\mathfrak{B} \Pi$-modules over $D \Gamma[\mathrm{HI}$, Lemma 1.2] there is a chain transformation from $D\left(\Gamma B_{*}\right)$ to $P_{*}$ over $1_{D \Gamma}$ which is unique up to homotopy and induces a homomorphism $\theta_{*}^{\mathfrak{B}}(\Gamma, A): \mathfrak{B}_{*}(\Gamma, A) \rightarrow$ $\operatorname{Tor}_{*}^{\mathfrak{B} \Pi}(D \Gamma, A)$. Since $\operatorname{Tor}_{n}^{\mathfrak{B} \Pi}(D \Gamma, A)=0$ for $n>0$ and $A$ projective, $\theta_{*}^{\mathfrak{B}}(\Gamma,-)$ is characterised as a $\delta$-morphism which is the identity in dimension 0 . Note that $\operatorname{Tor}_{*}^{\mathfrak{B} \Pi}(D \Gamma, A)$ (and hence $\theta_{*}^{\mathfrak{B}}(\Gamma, A)$ ) depends on $\Pi$ in a sense that $\mathfrak{B}_{*}(\Gamma, A)$ does not. Namely, let $\Gamma \rightarrow \Pi$ factorise as $\Gamma \rightarrow \bar{\Pi} \rightarrow \Pi$; then if $\bar{D} \Gamma=I \Gamma \otimes_{z \Gamma} \mathfrak{B} \bar{\Pi}, \operatorname{Tor}_{*}^{3 \bar{M}}(\bar{D} \Gamma, A)$ is not in general isomorphic to $\operatorname{Tor}_{*}^{\mathfrak{B M}}(D \Gamma, A)$, whereas $\mathfrak{B}_{n}(\Gamma, A)$ is the same if $A$ is regarded as a $\Pi$-module and homology is calculated in $(\mathfrak{B}, \Pi)$ or if $A$ is regarded as a $\bar{\Pi}$-module via $\bar{\Pi} \rightarrow \Pi$ and homology is calculated in $(\mathfrak{B}, \bar{\Pi})$. Our main concern will be with the problem of deciding when $\theta_{n}^{\mathfrak{Y}}(\Pi, A)$ is an isomorphism, as is the case when $\mathfrak{B}$ is the universal variety or is abelian.

Proposition 1.2. Let $\alpha: \Gamma_{0} \rightarrow \Gamma_{1}$ be a surjection in $(\mathfrak{B}, \Pi)$ and $A$ be a left $\mathfrak{B} \Pi-$ module. Put $K=\operatorname{ker} D \alpha$. Then there is a commutative diagram

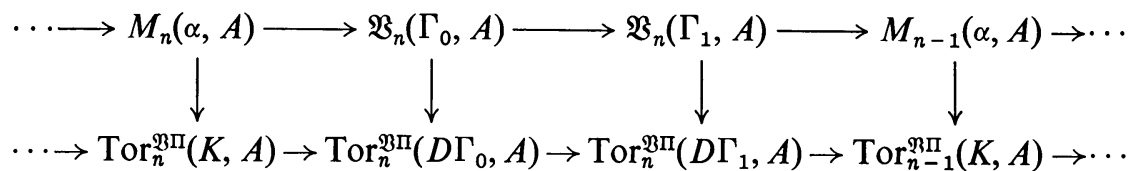


where the top row is (1.1), the bottom row is the homology sequence of $0 \rightarrow K \rightarrow D \Gamma_{0}$ $\rightarrow D \Gamma_{1} \rightarrow 0$, and $\mathfrak{B}_{n}\left(\Gamma_{i}, A\right) \rightarrow \operatorname{Tor}_{n}^{\mathfrak{B} \Pi}\left(D \Gamma_{i}, A\right)$ is $\theta_{n}^{\mathfrak{B}}\left(\Gamma_{i}, A\right)$. Moreover $M_{0}(\alpha, A)$ $=R / R^{\prime} \bigotimes_{\mathfrak{B} \Pi} A \rightarrow K \otimes A$ is $\beta \otimes A$ where $\beta: R / R^{\prime} \rightarrow K$ is determined by the commutativity of (1.3) in the case $A=\mathfrak{B} \Pi$.

(Note that $\beta$ appears in [HI, Lemmas 3.2 and 3.3].)

Proof. Let $0 \rightarrow \boldsymbol{P}^{\prime} \rightarrow \boldsymbol{P} \rightarrow \boldsymbol{P}^{\prime \prime} \rightarrow 0$ be a resolution of $0 \rightarrow K \rightarrow D \Gamma_{0} \rightarrow D \Gamma_{1} \rightarrow 0$. We have another exact sequence of complexes of projective modules over $0 \rightarrow K$ $\rightarrow D \Gamma_{0} \rightarrow D \Gamma_{1} \rightarrow 0$, namely $0 \rightarrow L(\alpha, \mathfrak{B} \Pi) \rightarrow D\left(\Gamma_{0} B\right) \rightarrow D\left(\Gamma_{1} B\right) \rightarrow 0$ (cf. Proposition 1.1). We can construct chain maps $f^{\prime}, f^{\prime \prime}$ from $L(\alpha, \mathfrak{B} \Pi)$ to $\boldsymbol{P}^{\prime}$ and from $D\left(\Gamma_{1} \boldsymbol{B}\right)$ to $\boldsymbol{P}^{\prime \prime}$ over $1_{K}$ and $1_{D \Gamma_{1}}$ respectively. Then by Northcott $[23, \S 5.7]$ there is a chain map $f$ from $D\left(\Gamma_{0} B\right)$ to $\boldsymbol{P}$ over $1_{D \Gamma_{0}}$ such that

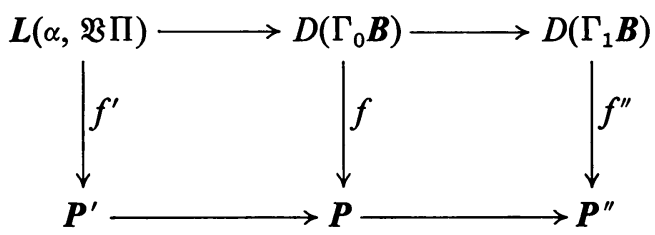

commutes. Tensoring with $A$ and taking homology gives (1.3). The last statement of the proposition follows from the right-exactness of $\otimes$.

Using the end of (1.3) we prove

Proposition 1.3. If $A$ is a left $\mathfrak{B} \Pi$-module, then there is an exact sequence

$\operatorname{Tor}_{2}^{\mathfrak{B} \Pi}(D \Pi, A) \longrightarrow \mathfrak{B}_{1}(\Pi, \mathfrak{B} \Pi) \otimes_{\mathfrak{B} \Pi} A \longrightarrow \mathfrak{B}_{1}(\Pi, A) \stackrel{\theta}{\longrightarrow} \operatorname{Tor}_{1}^{\mathfrak{B} \Pi}(D \Pi, A) \longrightarrow 0$, where $\theta=\theta_{1}^{\mathfrak{n}}(\Pi, A)$.

We shall add a term to this exact sequence later. The advantage of the present proof, apart from its simplicity, is that it helps to identify $\operatorname{ker} \theta_{1}^{\mathfrak{B}}(\Pi, A)$.

Proof. Let $1 \rightarrow R \rightarrow F \rightarrow \Pi \rightarrow 1$ be exact with $F \mathfrak{B}$-free, and $K$ be the kernel of $D F \rightarrow D \Pi$. Then there is a commutative diagram
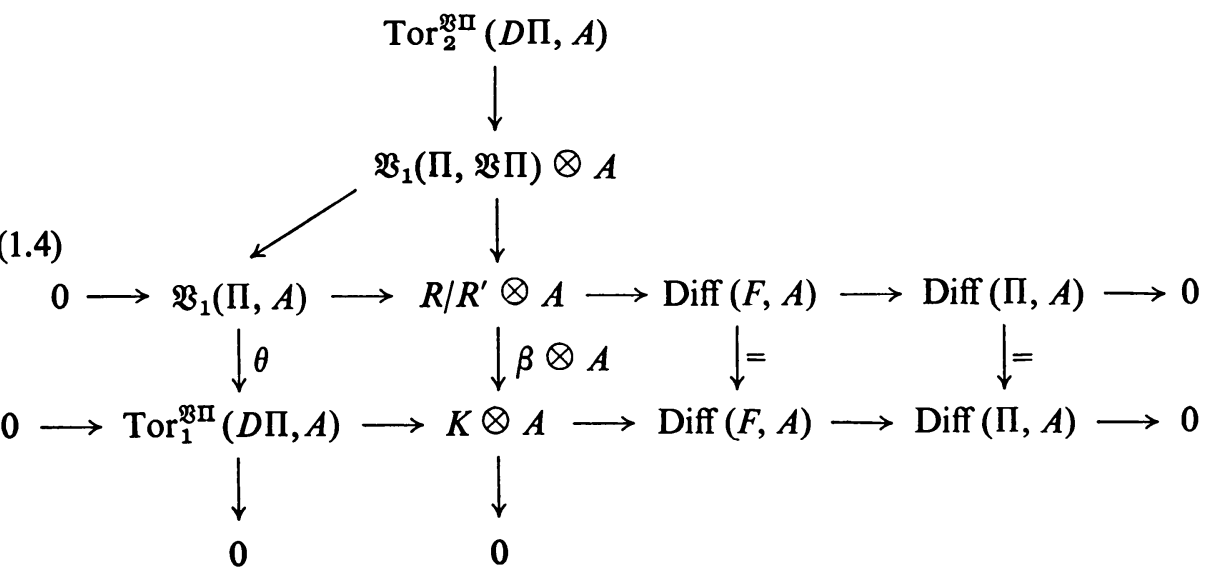
The lower part of the diagram comes from Proposition 1.2 with $\Gamma_{0}=F, \Gamma_{1}=\Pi$, bearing in mind that $\mathfrak{B}_{1}(F, A)=\operatorname{Tor}_{1}^{\mathfrak{M} \Pi}(D F, A)=0$. ( $D F$ is a free $\mathfrak{B} \Pi$-module, cf. [HI, Lemma 1.2].) The column is part of the homology sequence of the short exact sequence

$$
0 \longrightarrow \mathfrak{V}_{1}(\Pi, \mathfrak{B} \Pi) \longrightarrow R / R^{\prime} \stackrel{\beta}{\longrightarrow} K \longrightarrow 0, \quad \text { as } \operatorname{Tor}_{2}^{\mathfrak{B} \Pi}(D \Pi, A)=\operatorname{Tor}_{1}^{\mathfrak{B} \Pi}(K, A) .
$$

$\theta$ and $\beta \otimes A$ have "the same" kernel, so there is a unique homomorphism of $\mathfrak{B}_{1}(\Pi, \mathfrak{B} \Pi) \otimes A$ into $\mathfrak{B}_{1}(\Pi, A)$ which makes the diagram commute, and the sequence

$$
\operatorname{Tor}_{2}^{\mathfrak{B} \Pi}(D \Pi, A) \longrightarrow \mathfrak{B}_{1}(\Pi, \mathfrak{B} \Pi) \otimes A \longrightarrow \mathfrak{B}_{1}(\Pi, A) \longrightarrow \operatorname{Tor}_{1}^{\mathfrak{B} \Pi}(D \Pi, A) \longrightarrow 0
$$

is exact. This proves the proposition.

Since $\mathfrak{B}_{1}(\Pi, \mathfrak{B} \Pi$ ) is (isomorphic to) the kernel of $\beta$, and $\theta$ and $\beta \otimes A$ have "the same" kernel, one reads off from diagram (1.4), whose column is exact,

COROLlARY 1.4. $\theta_{1}^{\mathfrak{B}}(\Pi, A)$ is an isomorphism if and only if the homomorphism of $\mathfrak{B}_{1}(\Pi, \mathfrak{B} \Pi) \bigotimes_{\mathfrak{B} \Pi} A$ into $R / R^{\prime} \bigotimes_{\mathfrak{B} \Pi} A$ induced by the inclusion of $\mathfrak{B}_{1}(\Pi, \mathfrak{B} \Pi)$ into $R / R^{\prime}$ is the zero map.

Of course everything in this paragraph can be done as well in cohomology as in homology; in particular we get homomorphisms

$$
\theta_{\mathfrak{B}}^{n}(\Pi, A): \operatorname{Ext}_{\mathfrak{B}}^{n}(D \Pi, A) \longrightarrow \mathfrak{B}^{n}(\Pi, A) .
$$

2. Isomorphism in dimension 1. In [HI, §3] we showed that $\theta_{1}^{\mathfrak{P}}(\Pi, A)$ is an isomorphism for all $\mathfrak{B} \Pi$-modules $A$ if and only if $\theta_{\mathcal{P}}^{\mathfrak{P}}(\Pi, \mathfrak{B} \Pi)$ is an isomorphism; i.e. if and only if $\mathfrak{B}_{1}(\Pi, \mathfrak{B} \Pi)=0$; and that this is also equivalent to the condition that $\theta_{\mathfrak{B}}^{1}(\Pi, A)$ be an isomorphism for all $A$. We saw that these conditions are satisfied if $\Pi$ is cyclic, or of course if $\mathfrak{B}$ is the universal variety or is abelian, but is false if $\mathfrak{B}=\mathfrak{N}_{2}$ and $\Pi$ is Klein's four group. We show below that provided we restrict ourselves to modules with trivial action we have isomorphism in homology and in cohomology in a wider range of situations. In fact we know of no case in which $\theta_{1}^{\mathfrak{B}}(\Pi, A)$ and $\theta_{\mathfrak{B}}^{1}(\Pi, A)$ are not isomorphisms for $A$ a module with trivial action. The problem is akin to the dimension problem, and we shall need the fact that this has been solved in certain cases. If $\Pi$ is embedded in the natural way in $Z \Pi, d_{n} \Pi$, the $n$th dimension group of $\Pi$, is defined to be $\Pi \cap\left((I \Pi)^{n}+1\right)$. Then $d_{n} \Pi=\Pi_{(n)}$ for $n=2,3$, or if $\Pi$ is $\mathfrak{N}_{c}$-free for any $c, n>0$. See Passi [24].

LEMMA 2.1. Let $\mathfrak{B}$ be of exponent 0 . Then if $A$ is any $\Pi$-module on which $\Pi$ acts trivially, $A$ is a $\mathfrak{B} \Pi$-module; and if $\mathfrak{B}_{1}(\Pi, Z) \cong \operatorname{Tor}_{1}^{\mathfrak{B}}(D \Pi, Z)$ then

(i) $\mathfrak{B}_{1}(\Pi, A) \cong \operatorname{Tor}_{1}^{\mathfrak{M} \Pi}(D \Pi, A)$ and

(ii) $\mathfrak{B}^{1}(\Pi, A) \cong \operatorname{Ext}_{\mathfrak{B} \Pi}^{1}(D \Pi, A)$

for any $\Pi$-module $A$ with trivial action. 
Proof. The first statement is clear (see [HI, the beginning of $\S 4]$ ). By universal coefficients [HI, Lemma 4.1], if $\Pi$ acts trivially on $A$,

$$
\mathfrak{B}_{1}(\Pi, A) \cong \mathfrak{B}_{1}(\Pi, Z) \otimes A \oplus \operatorname{Tor}_{1}^{Z}\left(\Pi / \Pi^{\prime}, A\right) .
$$

(Note that Diff $(\Pi, Z)=\Pi / \Pi^{\prime}$.) Similarly

$$
\operatorname{Tor}_{1}^{\mathfrak{M}}(D \Pi, A) \cong \operatorname{Tor}_{1}^{\mathfrak{M} \Pi}(D \Pi, Z) \otimes A \oplus \operatorname{Tor}_{1}^{Z}\left(\Pi / \Pi^{\prime}, A\right)
$$

Hence if $\mathfrak{B}_{1}(\Pi, Z) \cong \operatorname{Tor}_{1}^{\mathfrak{B} \Pi}(D \Pi, Z)$, (i) follows, and similarly (ii).

In practice the varieties we discuss will all be of exponent zero. Thus the lemma shows that as far as modules with trivial action are concerned we may restrict ourselves to proving that $\theta_{1}^{\mathfrak{B}}(\Pi, Z)$ is an isomorphism.

THEOREM 2.2. If $\mathfrak{B}=\mathfrak{N}_{2}$, or if $\mathfrak{B}$ contains $\mathfrak{N}_{2}$ and $\Pi$ is abelian, then $\theta_{1}^{\mathfrak{P}}(\Pi, Z)$ is an isomorphism.

Proof. Let $1 \rightarrow R \rightarrow F \stackrel{\alpha}{\rightarrow} \Pi \rightarrow 1$ be exact with $F \mathfrak{B}$-freely generated by the set $\mathfrak{x}$. By Corollary 1.4 it is enough to prove that $\mathfrak{B}_{1}(\Pi, \mathfrak{B} \Pi) \otimes_{\mathfrak{B H}} Z \rightarrow R / R^{\prime} \otimes_{\mathfrak{B} \Pi} Z$ $=R /[R, F]$ is the zero map. By [HI, Lemma 3.3], $\mathfrak{B}_{1}(\Pi, \mathfrak{B} \Pi)=\operatorname{ker} \beta$ is the subgroup of $R / R^{\prime}$ consisting of elements $r R^{\prime}$ such that $\partial^{\alpha} r / \partial x=0$ for all $x \in \mathfrak{x}$. Let $r \in R$ satisfy these equations. We shall prove that $r \in[R, F]$, and this will prove the theorem. From diagram (1.4) one sees that $r[R, F]$ is in the kernel of $R /[R, F]$ $=R / R^{\prime} \otimes Z \rightarrow \operatorname{Diff}(F, Z)=F / F^{\prime}$. Thus $r \in R \cap F^{\prime}$. Put $\mathfrak{x}=\left\{x_{i} \mid i \in I\right\}$, and $x_{i} \alpha=a_{i}$ for all $i \in I$. We may assume that $r$ has been chosen in $r[R, F]$ to involve $x_{i}$ for the least possible number of values of $i$ in $I$. Moreover commutators of weight 3 may be discarded, since if $\Pi$ is abelian they lie in $[R, F]$, and if $\mathfrak{B}=\mathfrak{N}_{2}$ they represent the identity. Assume now that $r \neq 1$. Then if $x_{1}$ is involved in $r$, discarding commutators of weight 3 gives $r=c\left[x_{1}, w\right]^{n}$, where $w \in F$ and $c$ is a product of commutators not involving $x_{1}$. Also, since $\left[x_{1}, w\right]^{n} \equiv\left[x_{1}, w^{n}\right] \bmod F_{(3)}$, we may assume $n=1 . \partial^{\alpha} r / \partial x_{1}=0$ now gives immediately the condition that $1-\left(w^{-1} \alpha\right)^{a_{1}}$ represent zero in $\mathfrak{B} \Pi$. Since $\mathfrak{B} \supseteq \mathfrak{R}_{2}$ this implies by [HI, Proposition 1.3] that $1-\left(w^{-1} \alpha\right)^{a_{1}} \in(I \Pi)^{2}$. Hence, by the dimension property, $\left(w^{-1} \alpha\right)^{a_{1}} \in \Pi^{\prime}$, so $w \alpha \in \Pi^{\prime}$. .If now $\Pi$ is abelian $w \in R$, so $\left[x_{1}, w\right] \in[R, F]$, and dropping this term from $r$ gives a contradiction since $x_{1}$ is no longer involved. On the other hand if $\mathfrak{B}=\mathfrak{N}_{2}$, since $w \alpha \in \Pi^{\prime}, w$ is a product of commutators and an element of $R$, so again $\left[x_{1}, w\right] \in[R, F]$ and we have a contradiction as before.

In fact the second case considered in the theorem can be deduced from the first using

Proposition 2.3. If $\mathfrak{B}$ contains [్, var $\Pi$ ], or if $\Pi$ is finite and $\mathfrak{B}$ is of exponent 0 and contains a covering group of $\Pi$, then $\mathfrak{B}_{1}(\Pi, Z)=H_{2}(\Pi, Z)$. Moreover if $\theta_{1}^{\mathfrak{P}}(\Pi, Z)$ is an isomorphism then so is $\theta_{\mathfrak{P}}^{\mathfrak{P}}(\Pi, Z)$ for any variety $\mathfrak{W}$ containing $\mathfrak{B}$. 
Proof. Let $1 \rightarrow R \rightarrow F \rightarrow \Pi \rightarrow 1$ be exact with $F \mathfrak{W}$-free. This gives rise to a commutative diagram

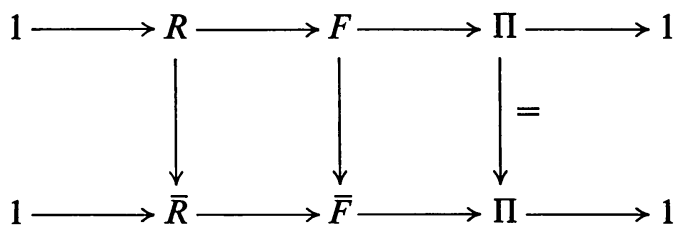

where $\bar{F}=F / \mathfrak{B}\langle F\rangle$ and $F \rightarrow \bar{F}$ is the natural surjection. This induces a surjection

$$
\omega: \mathfrak{W}_{1}(\Pi, Z)=R \cap F^{\prime} /[R, F] \rightarrow \bar{R} \cap \bar{F}^{\prime} /[\bar{R}, \bar{F}]=\mathfrak{B}_{1}(\Pi, Z)
$$

(see [HI, §4]). Now suppose that $r[R, F]$ is in the kernel of $\omega$, and that $\mathfrak{B}$ contains [ङ, var $\Pi$ ]. Then $r$ may be taken as a product of commutators $[x, w]$ and their inverses, where $w$ is a law in var $\Pi$. Thus $r \in[R, F]$, so $\omega$ is an isomorphism. That $\omega$ is an isomorphism in the second case considered in the proposition follows immediately from [HI, Theorem 4.2]. Putting $\mathfrak{W}$ the universal variety now gives the first statement of the proposition.

Writing $D_{\mathfrak{Y}} \Pi=D \Pi=I \Pi \otimes_{z \Pi} \mathfrak{B} \Pi$, and similarly for $D_{\mathfrak{B}} \Pi$, so that $D_{\mathfrak{B}} \Pi$ $=D_{\mathfrak{B}} \Pi \otimes_{\mathfrak{W} \Pi} \mathfrak{B} \Pi$, we have a diagram

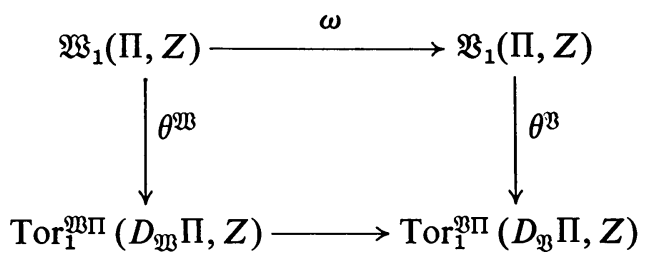

where $\theta^{\mathfrak{B}}=\theta^{\mathfrak{B}}(\Pi, Z)$ and similarly for $\theta^{\mathfrak{P}}$, and $\operatorname{Tor}_{1}^{\mathfrak{P} \Pi}\left(D_{\mathfrak{B}} \Pi, Z\right) \rightarrow \operatorname{Tor}_{1}^{\mathfrak{B} \Pi}\left(D_{\mathfrak{B}} \Pi, Z\right)$ is the "change of rings" homomorphism.

It is easy to see in various ways that this diagram commutes: we shall consider these "change of variety" homomorphisms in more detail in a later paper. Now under the hypotheses of the proposition we have seen that $\omega$ is an isomorphism, as is $\theta^{\mathfrak{B}}$ by assumption. But $\theta^{\mathfrak{W}}$ is a surjection. It follows that $\theta^{\mathfrak{M}}$ is an isomorphism, and the proposition is proved.

The next result allows us to deduce the first case of Theorem 2.2 from the second.

Proposition 2.4. Let $\mathfrak{B}=[\mathfrak{E}, \mathfrak{u}]$ and assume that $\theta_{1}^{\mathfrak{P}}(\Pi / \mathfrak{u}\langle\Pi\rangle, Z)$ is an isomorphism. Then so is $\theta_{1}^{\mathfrak{B}}(\Pi, Z)$.

Proof. Take $1 \rightarrow R \rightarrow F \stackrel{\alpha}{\rightarrow} \Pi \rightarrow 1$ exact with $F \mathfrak{B}$-free. Let $r \in R \cap F^{\prime}$ represent an element of $\operatorname{ker} \theta_{1}^{\mathfrak{M}}(\Pi, Z)$. Then $\partial^{\alpha} r / \partial x=0$ for every canonical free generator $x$ of $F$. Put $\bar{\alpha}=F \rightarrow \Pi \rightarrow \Pi / \mathfrak{u}\langle\Pi\rangle$, and $S=\operatorname{ker} \bar{\alpha}$. Then a fortiori $r \in S \cap F^{\prime}$ and $\partial^{\bar{\alpha}} r / \partial x=0$ for every $\mathfrak{B}$-free generator $x$ of $F$. But $\theta_{1}^{\mathfrak{S}}(\Pi / \mathfrak{u}\langle\Pi\rangle, Z)$ is an isomorphism, so $r \in[S, F]$. However, $S$ is generated by $R$ and $\mathfrak{u}\langle F\rangle$, and $F$ centralizes $\mathfrak{H}\langle F\rangle$. Hence $[S, F]=[R, F]$, and so $r \in[R, F]$. This proves the proposition. 
In particular, to replace $\mathfrak{N}_{2}$ by $\mathfrak{N}_{3}$ in Theorem 2.2 it is enough to look at groups of class exactly 2 . The next result is a partial converse to the last one, except that the restriction to modules with trivial $\Pi$-action can be dropped.

Proposition 2.5. If $\mathfrak{B}=\mathfrak{R}_{c}$ and $\Pi / \Pi_{(c)}$ is $\mathfrak{R}_{c-1}$-free, then $\theta_{1}^{\mathfrak{N}}(\Pi, A)$ is an isomorphism for any $\mathfrak{B} \Pi$-module $A$.

For various equivalent formulations of the conclusion, see [HI, Lemma 3.2].

Proof. Let $1 \rightarrow R \rightarrow F \stackrel{\alpha}{\rightarrow} \Pi \rightarrow 1$ be exact, with $F \mathfrak{N}_{c}$-free, and $\alpha$ mapping the $\mathfrak{N}_{c}$-free generators of $F$ to representatives of the $\mathfrak{R}_{c-1}$-free generators of $\Pi / \Pi_{(c)}$. Then $R$ is abelian and is generated by commutators of weight $c$. Let $r \in R, r \neq 1$. $F$, being $\mathfrak{R}_{c}$-free, enjoys the dimension property, so $1-r \notin(I F)^{c+1}$. But, as was observed in [HI, §3], $1-r=\sum\left(1-x_{i}\right) \partial r / \partial x_{i}$, the sum being taken over the $\mathfrak{N}_{c}$-free generators of $F$. Hence $\partial r / \partial x_{i} \notin(I F)^{c}$ for some $i$. By [HI, Lemma 3.3] it is enough to prove that $\partial^{\alpha} r / \partial x_{i} \neq 0$ for some $i$; assume then that $\partial^{\alpha} r / \partial x_{i}=0$ for all $i$. Then $\partial r / \partial x_{i}=s+k$ where $s \in(I F)^{c}$ and $k \in \operatorname{ker}(I F \rightarrow I \Pi)=\operatorname{ker}(Z F \rightarrow Z \Pi)$. Now $\operatorname{ker}(Z F \rightarrow Z \Pi)$ is generated by $\{1-w \mid w \in R\}$, and since $R$ is generated by commutators of weight $c, 1-w \in(I F)^{c}$ for all $w \in R$. Hence $\partial r / \partial x_{i} \in(I F)^{c}$ for all $i$, contradiction.

Combining Propositions 2.3, 2.4 and 2.5 gives various corollaries which we refrain from stating.

3. Universal coefficients. As André [1], Barr and Beck [4], Ulmer [20] and Rinehart [13] have done, we construct a spectral sequence

$$
\operatorname{Tor}_{p}^{\mathfrak{B} \Pi}\left(\mathfrak{B}_{q}(\Pi, \mathfrak{B} \Pi), A\right) \underset{p}{\Rightarrow} \mathfrak{B}_{n}(\Pi, A)
$$

where $A$ is a $\mathfrak{B} \Pi$-module. The point of our treatment is that we calculate the edge effects so that we can calculate the homomorphisms in the exact sequence of terms of low degree. This stretches the exact sequence of Proposition 1.3 by one term, and gives at once a case when $\theta_{2}^{\mathfrak{B}}(\Pi, Z)$ is not an isomorphism.

THEOREM 3.1. For every projective resolution $\ldots \rightarrow P_{0} A \stackrel{\varepsilon}{\rightarrow} A$ of the left $\mathfrak{B} \Pi-$ module $A$, there is a spectral sequence

$$
\operatorname{Tor}_{p}^{\mathfrak{B} \Pi}\left(\mathfrak{B}_{q}(\Pi, \mathfrak{B} \Pi), A\right) \underset{p}{\Rightarrow} \mathfrak{B}_{n}(\Pi, A),
$$

with edge effects as follows. $\mathfrak{B}_{n}(\Pi, A) \rightarrow \operatorname{Tor}_{n}^{\mathfrak{B} \Pi}(D \Pi, A)=E_{n 0}^{2}$ is the homomorphism $\theta_{n}^{\mathfrak{Q}}(\Pi, A)$ of the previous paragraph.

$$
\mathfrak{B}_{n}\left(\Pi, P_{0} A\right)=E_{0 n}^{1} \rightarrow \mathfrak{B}_{n}(\Pi, A) \text { is } \mathfrak{B}_{n}(\Pi, \varepsilon) .
$$

Proof. Construct a simplicial resolution of $\Pi$ by $\mathfrak{B}$-free groups; for example the Barr-Beck resolution. Applying the functor $D$ gives an augmented complex of free $\mathfrak{B} \Pi$-modules $Q \Pi \rightarrow D \Pi \rightarrow 0$. Then for any $\mathfrak{B} \Pi$-module $A^{\prime}, \mathfrak{B}_{*}\left(\Pi, A^{\prime}\right)$ is the homology of $Q \Pi \otimes_{\mathfrak{B} \Pi} A^{\prime}$. Construct the first quadrant double complex $T_{p q}$ $=Q_{q} \Pi \otimes_{\mathfrak{B} \Pi} P_{p} A$. (We shall follow the notation of Cartan and Eilenberg [6], but 
whether the differentials of $\boldsymbol{T}$ are chosen to commute or anticommute will be of no significance.) Fixing $p$ and taking homology gives

$$
\mathfrak{B}_{q}\left(\Pi, P_{p} A\right)=\mathfrak{B}_{q}(\Pi, \mathfrak{B} \Pi) \bigotimes_{\mathfrak{B} \Pi} P_{p} A .
$$

Taking homology again gives $\operatorname{Tor}_{p}^{\mathfrak{B} \Pi}\left(\mathfrak{B}_{q}(\Pi, \mathfrak{B} \Pi), A\right)$. So for the first spectral sequence we have $E_{p q}^{2}=I_{p q}^{2}=\operatorname{Tor}_{p}^{\mathfrak{B} \Pi}\left(\mathfrak{B}_{q}(\Pi, \mathfrak{B} \Pi), A\right)$. For the second spectral sequence, fixing $q$ and taking homology in $T$ gives $\operatorname{Tor}_{p}^{\mathfrak{B} \Pi}\left(Q_{q} \Pi, A\right)=Q_{q} \Pi \otimes_{\mathfrak{B} \Pi} A$ for $p=0$ and is zero for $p>0$. Hence the second spectral sequence collapses to give $\mathfrak{B}_{n}(\Pi, A)$. It remains to calculate the edge effects. For this purpose we use Mac Lane [22, Theorem XI, 4.4] which states that if $I_{p q}^{r}$ is the first spectral sequence of the first quadrant double complex $S_{p q}$ then the edge effects are given by $I_{0 n}^{1}=H_{n} S_{0 *} \rightarrow H_{n} S$ induced by the inclusion of $S_{0 *}$ in $S$, and $H_{n} S \rightarrow H_{n}(S / M)$ $=I_{n 0}^{2}$ induced by the projection of $S$ on $S / M$, where $M$ is the subcomplex of $S$ given by

$$
M_{n}=\sum_{p+q=n ; q>0} S_{p q} \cup \partial \sum_{p+q=n+1 ; q>0} S_{p q} .
$$

We apply this first to the second spectral sequence; that is with $S=\boldsymbol{T}$ transposed. In this case $S / M$ is chain isomorphic to $Q \Pi \otimes_{\mathfrak{B} \Pi} A$ via the isomorphism of coker $\left(P_{1} A \rightarrow P_{0} A\right)$ onto $A$ induced by $\varepsilon$. Hence we have an isomorphism $\omega$ of $H_{*} T$ onto $\mathfrak{B}_{*}(\Pi, A)$. Now looking at the first spectral sequence and applying the first part of the above theorem gives us the homomorphism $\zeta$ in the diagram

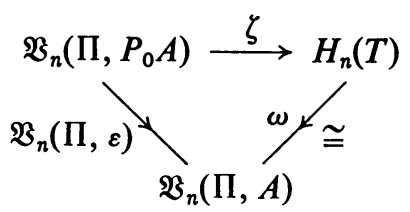

which is clearly commutative. This identifies the edge homomorphism on the fibre. Now let $\bar{Q} \Pi \rightarrow D \Pi \rightarrow 0$ be a projective resolution of $D \Pi$. Then there is a chain map $f: \boldsymbol{Q} \Pi \rightarrow \overline{\boldsymbol{Q}} \Pi$ over $1_{\mathfrak{B} \Pi}$, unique up to homotopy. Let $\overline{\boldsymbol{T}}=\overline{\boldsymbol{Q}} \Pi \otimes_{\mathfrak{B} \Pi} \boldsymbol{P} A$ with the same sign convention as before. Then $f$ induces a chain map $F: T \rightarrow \overline{\boldsymbol{T}}$. Now let $\boldsymbol{M}_{1}, \boldsymbol{M}_{2}, \overline{\boldsymbol{M}}_{1}$ and $\overline{\boldsymbol{M}}_{2}$ correspond to $\boldsymbol{M}$ in the theorem quoted above, where $\boldsymbol{S}$ is taken as $T, T$ transposed, $\bar{T}$ and $\bar{T}$ transposed respectively. Then $T / M_{1}$ and $\overline{\boldsymbol{T}} / \overline{\boldsymbol{M}}_{1}$ are naturally isomorphic to $D \Pi \otimes_{\mathfrak{B} \Pi} \boldsymbol{P} A, \boldsymbol{T} / \boldsymbol{M}_{2}$ to $\boldsymbol{Q} \Pi \otimes_{\mathfrak{B} \Pi} A$, and $\overline{\boldsymbol{T}} / \overline{\boldsymbol{M}}_{2}$ to $\bar{Q} \Pi \otimes_{\mathfrak{B} \Pi} A$. Using the natural identifications, $F$ induces a commutative diagram

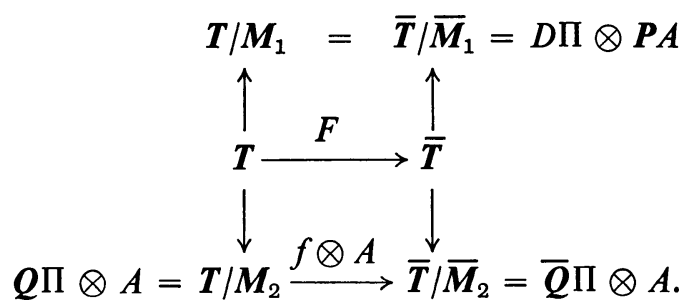


Taking homology, the usual identifications give

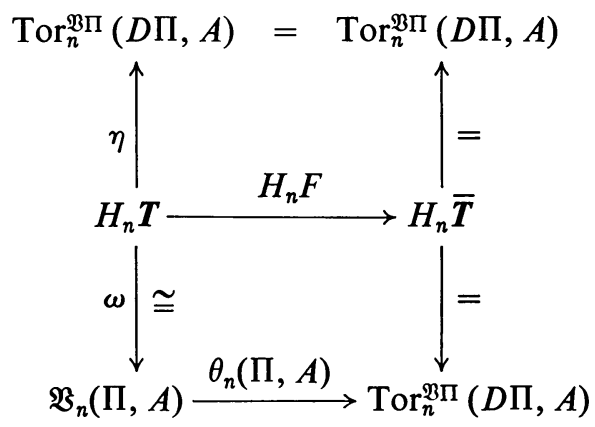

where $\eta$ is the base homomorphism and $\omega$ is the isomorphism of the first part. This completes the proof of the theorem.

COROLlaRY 3.2. There is an exact sequence

$$
\begin{aligned}
\mathfrak{B}_{2}(\Pi, A) \stackrel{\theta_{2}}{\longrightarrow} \operatorname{Tor}_{2}^{\mathfrak{P} \Pi}(D \Pi, A) \longrightarrow \mathfrak{B}_{1}(\Pi, \mathfrak{B} \Pi) \otimes_{\mathfrak{B} \Pi} A & \\
& \longrightarrow \mathfrak{B}_{1}(\Pi, A) \stackrel{\theta_{1}}{\longrightarrow} \operatorname{Tor}_{1}^{\mathfrak{Y} \Pi}(D \Pi, A) \longrightarrow 0
\end{aligned}
$$

where $\theta_{i}=\theta_{i}^{\mathfrak{P}}(\Pi, A)$, and $\mathfrak{B}_{1}(\Pi, \mathfrak{B} \Pi) \otimes_{\mathfrak{B} \Pi} A \rightarrow \mathfrak{B}_{1}(\Pi, A)$ is as in Proposition 1.3.

No doubt the homomorphism $\operatorname{Tor}_{2}^{\mathfrak{B} \Pi}(D \Pi, A) \rightarrow \mathfrak{B}_{1}(\Pi, \mathfrak{B} \Pi) \otimes_{\mathfrak{B} \Pi} A$ also agrees with the corresponding homomorphism of Proposition 1.3 but this is of no significance, whereas $\mathfrak{B}_{1}(\Pi, \mathfrak{B} \Pi) \bigotimes_{\mathfrak{B} \Pi} A \rightarrow \mathfrak{V}_{1}(\Pi, A)$ is well under control (see diagram (1.4) and Corollary 1.4).

Proof. Taking the exact sequence of terms of low degree of the spectral sequence, it is enough to check that the homomorphisms of $\mathfrak{V}_{1}(\Pi, \mathfrak{B} \Pi) \bigotimes_{\mathfrak{B} \Pi} A$ into $\mathfrak{B}_{1}(\Pi, A)$ in the two exact sequences coincide. To that end consider the diagram

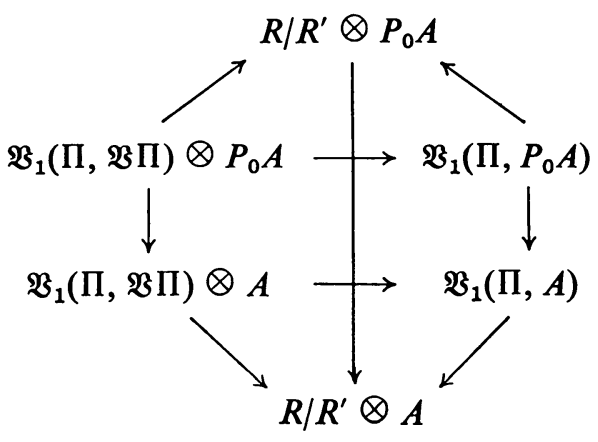

Here $\mathfrak{B}_{1}\left(\Pi, P_{0} A\right) \rightarrow R / R^{\prime} \bigotimes_{\mathfrak{B I}} P_{0} A$ and $\mathfrak{B}_{1}(\Pi, A) \rightarrow R / R^{\prime} \bigotimes_{\mathfrak{B} \Pi} A$ are as in Rinehart's exact sequence, $\mathfrak{B}_{1}(\Pi, \mathfrak{B} \Pi) \otimes_{\mathfrak{B} \Pi} P_{0} A \rightarrow \mathfrak{B}_{1}\left(\Pi, P_{0} A\right)$ is the natural isomorphism, $E_{01}^{2}=\mathfrak{B}_{1}(\Pi, \mathfrak{B} \Pi) \otimes_{\mathfrak{B} \Pi} A \rightarrow \mathfrak{B}_{1}(\Pi, A)$ is the fibre homomorphism, and the rest are induced in various ways by the inclusion of $\mathfrak{B}_{1}(\Pi, \mathfrak{B} \Pi)$ in $R / R^{\prime}$ and the 
surjection $\varepsilon: P_{0} A \rightarrow A$. The square involving $\mathfrak{B}_{1}(\Pi, \mathfrak{B} \Pi) \bigotimes_{\mathfrak{B} \Pi} P_{0} A \rightarrow \mathfrak{B}_{1}\left(\Pi, P_{0} A\right)$ commutes easily. The square involving $\mathfrak{B}_{1}(\Pi, A) \rightarrow R / R^{\prime} \bigotimes_{\mathfrak{B} \Pi} A$ commutes since Rinehart's exact sequence is functorial in the module, and the third square and the triangle involving $R / R^{\prime} \otimes_{\mathfrak{B} \Pi} P_{0} A$ commute trivially. Hence, since $\mathfrak{B}_{1}(\Pi, \mathfrak{B} \Pi) \otimes_{\varepsilon}$ is a surjection, the other triangle commutes. This identifies the edge homomorphism $\mathfrak{B}_{1}(\Pi, \mathfrak{B} \Pi) \otimes_{\mathfrak{B} \Pi} A \rightarrow \mathfrak{B}_{1}(\Pi, A)$ with the corresponding homomorphism in Proposition 1.3.

EXAMPLE. If $\mathfrak{B}=\mathfrak{N}_{2}$ and $\Pi$ is Klein's four group, then $\mathfrak{V}_{1}(\Pi, \mathfrak{B} \Pi)$ has exactly two elements, as can be seen from the proof of [HI, Theorem 3.5]. Hence the action of $\Pi$ on $\mathfrak{B}_{1}(\Pi, \mathfrak{B} \Pi)$ is trivial, and $\mathfrak{B}_{1}(\Pi, \mathfrak{B} \Pi) \otimes_{\mathfrak{B}_{\Pi}} Z \cong \mathfrak{B}_{1}(\Pi, \mathfrak{B} \Pi)$. Since by Theorem $2.2 \theta_{1}^{\mathfrak{B}}(\Pi, Z)$ is an isomorphism in this case, it follows that $\theta_{2}^{\mathfrak{P}}(\Pi, Z)$ is not. Of course the possibility remains that $\mathfrak{B}_{2}(\Pi, Z)$ and $\operatorname{Tor}_{2}^{\mathfrak{B} \Pi}(D \Pi, Z)$ are isomorphic. However we shall show in a later paper that these groups are widely different in some varieties.

Of course everything in this paragraph has an analogue in cohomology. We omit the details.

\section{BIBLIOGRAPHY}

21. C. R. Leedham-Green, Homology in varieties of groups. I, Trans. Amer. Math. Soc. 162 (1971), 1-14.

22. S. Mac Lane, Homology, Die Grundlehren der math. Wissenschaften, Band 114, Academic Press, New York; Springer-Verlag, Berlin, 1963. MR 28 \#122.

23. D. G. Northcott, An introduction to homological algebra, Cambridge Univ. Press, New York, 1960. MR 22 \#9523.

24. I. B. S. Passi, Dimension subgroups, J. Algebra 9 (1968), 152-182. MR 38 \#242.

Queen Mary College, London, England 